\title{
Density Functional Calculation \\ of Dioxygen Adsorption at Complexes \\ of Ceria Nanoparticle with Atoms, Trimers \\ and Tetramers of Silver
}

\author{
Vladimir A. Nasluzova, Konstantin M. Neyman ${ }^{\mathrm{b}, \mathrm{c}}$, Aleksey M. Shor \\ Svetlana S. Laletina ${ }^{a}$ and Elena A. Ivanova-Shor ${ }^{* a}$ \\ anstitute of Chemistry and Chemical Technology SB RAS \\ FRC "Krasnoyarsk Science Center SB RAS" \\ 50/24, Akademgorodok, Krasnoyarsk, 660036, Russia \\ ${ }^{b}$ Departament de Ciència de Materials i Química Física \\ Universitat de Barcelona \\ 1 C/Martí i Franquès, 08028, Barcelona, Spain \\ 'ICREA (Institució Catalana de Recerca i Estudis Avançats) \\ 23 Pg. Lluís Companys, 08010, Barcelona, Spain
}

Received 12.05.2016, received in revised form 24.07.2016, accepted 04.09.2016

\begin{abstract}
Molecular adsorption (MA) and dissociative adsorption (DA) of $\mathrm{O}_{2}$ on complexes of a model nanoparticle $\mathrm{Ce}_{21} \mathrm{O}_{42}$ (NP) with an atom and small clusters of silver have been addressed. According to results of density functional calculations formation energies of such systems (with respect to the non-interacting $\mathrm{NP}, \mathrm{Ag}_{n}$ and $\left.\mathrm{O}_{2}\right)$ are calculated to be 2.0-4.4 eV. $\mathrm{O}_{2}$ adsorption energy $\left(E_{a d}\left(\mathrm{O}_{2}\right)\right)$ in the lowest-energy atomic complex with $\mathrm{O}_{2}$ in a bridging position between Ce ion and Ag bound on the \{111\} nanofacet (AgOO\{Ce\}-complex) is as high as $\sim 1.3 \mathrm{eV}$. $\mathrm{Ag}_{n} \mathrm{OO}\{\mathrm{Ce}\}$ - and $\mathrm{OAg}_{n} \mathrm{O}\{\mathrm{Ce}\}$-bridging structures are formed in MA and DA lowest-energy complexes of $\mathrm{Ag}_{3}$ on $\{100\}$ and $\{111\}$ as well as $\mathrm{Ag}_{4}$ on $\{100\}$ nanofacets. Bonding characteristics of the $\mathrm{Ag}_{n} \mathrm{OO}\{\mathrm{C}\}^{-}$-structures match those for stable superoxo groups $\mathrm{O}_{2}^{-} \cdot E_{\text {ad }}\left(\mathrm{O}_{2}\right)$ of the lowest-energy $\mathrm{MA}$ and DA complexes of $\mathrm{O}_{2}$ with Ag clusters are in the range of 0.5-1.1 and 1.4-2.0 eV, respectively. Activation energies for $\mathrm{O}_{2}$ dissociation in $\mathrm{Ag}_{n} \mathrm{OO}\{\mathrm{Ce}\}$ complexes $(n=3,4)$ calculated to be $1.5-2.1 \mathrm{eV}$ indicate low rates of these oxidation rearrangements.
\end{abstract}

Keywords: ceria, nanoparticles, silver clusters, oxygen molecule, adsorption, dissociation, density functional method.

DOI: $10.17516 / 1998-2836-2016-9-3-281-295$.

(C) Siberian Federal University. All rights reserved

* Corresponding author E-mail address: sekr@icct.ru 


\title{
Расчеты адсорбции кислорода
}

\section{на комплексах наночастицы оксида церия с атомами, тримерами и тетрамерами серебра \\ методом функционала плотности}

\author{
В.А. Наслузов ${ }^{a}$, К.М. Нейман ${ }^{6, \text { в }}$ \\ А.М. Шор ${ }^{\text {a }, ~ С . С . ~ Л а л е т и н а ~}{ }^{\text {a }}$ Е.А. Иванова-Шора \\ ${ }^{a}$ Институт химии и химической технологии СО РАН \\ ФИЦ «Красноярский научный иентр СО РАН» \\ Россия, 660036, Красноярск, Академгородок, 50/24 \\ ${ }^{6}$ Кафедра материаловедения и физической химии, \\ Университет Барселоны, \\ с/Марти и Франкес 1, 08028 Барселона, Испания \\ ${ }^{6}$ Научный центр прогрессивных исследований \\ пр. Льюиса Компаниса 23, 08010 Барселона, Испания
}

В статье рассмотрены продукты молекулярной адсорбциии (МА) и диссоциативной адсорбции (ДА) молекулы $\mathrm{O}_{2}$ на комплексах модельной наночастицы $\mathrm{Ce}_{21} \mathrm{O}_{42}$ (NP) с атомом и небольшими кластерами серебра. Согласно данным расчетов методом функиионала плотности энергии образования координированных с $O_{2}$ мономеров, тримеров и тетрамеров серебра (относительно невзаимодействующих $\mathrm{NP}, \mathrm{Ag}_{n}$ и $\mathrm{O}_{2}$ ) составляют 2.0-4.4 эВ. Энергия адсорбичии молекуль $\mathrm{O}_{2}\left(E_{a d}\left(\mathrm{O}_{2}\right)\right)$ в наиболее стабильном атомарном $\mathrm{AgOO}\{\mathrm{Ce}\}$-комплексе с молекулой $\mathrm{O}_{2}$ в мостиковом положении между атомами Се и $\mathrm{Ag}$ на поверхности нанограни $\{111\}$ достигает $\sim 1.3$ эB. $\mathrm{Ag}_{n} \mathrm{OO}\{\mathrm{Ce}\}-$ и $\mathrm{OAg}_{n} \mathrm{O}\{\mathrm{Ce}\}$-мостиковые структуры образуются и в наиболее стабильных изомерах МА- и ДА-комплексов $\mathrm{Ag}_{3}$ на \{100\} и \{111\} и $\mathrm{Ag}_{4}$ на \{100\} наногранях. Характеристики химической связи $\mathrm{O}-\mathrm{O}$ в $\mathrm{Ag}_{n} \mathrm{OO}\{\mathrm{Ce}\}$-структурах указывают на образование стабильных супероксидных группировок $\mathrm{O}_{2}^{-} . E_{a d}\left(\mathrm{O}_{2}\right)$ для наиболее стабильных МА и ДА комплексов на кластерах серебра и ограничиваются величинами 0.5-1.1 и 1.4-2.0 эВ

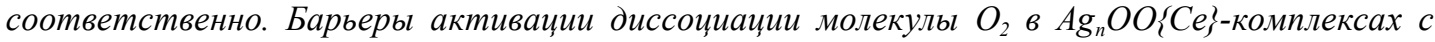
$n=3$ и 4 составляют 1.5-2.1 эB, что свидетельствует о низких скоростях протекания данных окислительных перегруппировок.

Ключевые слова: оксид иерия, наночастищы, кластеры серебра, молекула кислорода, адсорбиия, диссоциация, метод функциионала плотности.

\section{Введение}

Взаимодействие ультрадисперсных форм серебра с поверхностями оксидных подложек приводит к образованию изолированных $\mathrm{Ag}^{+}$катионных комплексов, $\mathrm{Ag}_{n}^{\delta+}$ кластеров, островков металлической и окисной $\mathrm{Ag}_{2} \mathrm{O}$ фаз [1-5]. Данные системы известны в качестве катализаторов реакций низкотемпературного окисления $[1,3,5-7]$. В целом, активность данных 
катализаторов определяется характеристиками тех или иных поверхностных комплексов $\mathrm{O}_{2}$ (см. [8, 9] с источниками, [3, 5, 7, 10-12]). В частности на поверхностях металлической фазы предпочтительным оказывается образование пероксидных $O_{2}^{2-}$ групп, и их диссоциации препятствуют сравнительно небольшие энергии активации в 0.6-1.1 эВ [10, 13].

Специальный интерес представляет взаимодействие молекулы $\mathrm{O}_{2}$ с предельно диспергированными формами серебра (атомы и кластеры), для которых ожидается критическая зависимость свойств от размерных и морфологических характеристик металлического остова $\mathrm{Ag}_{\mathrm{n}}$ (для кластеров) и от особенностей химической связи с кристаллической решеткой оксида $[12,14]$. В отличие от поверхности протяженных систем можно ожидать, что на поверхности субнаноразмерных кластеров будут предпочтительно стабилизироваться молекулярные формы кислорода [9, 13].

Ранее довольно детально исследовалось взаимодействие молекулы $\mathrm{O}_{2}$ с газофазными субнаноразмерными частицами $\operatorname{Ag}_{n}$ (см. [15] с источниками, $\left.[13,16,17]\right)$. При этом энергия активации диссоциации $\mathrm{O}_{2}$ на небольших частицах $\mathrm{Ag}_{\mathrm{n}}$ была рассчитана большей, чем для диссоциации $\mathrm{O}_{2}$ на протяженных поверхностях [13]. В ряде теоретических работ исследовалось взаимодействие молекулы $\mathrm{O}_{2}$ с субнаноразмерными кластерами и атомами серебра, закрепленными на оксидных подложках $[10,12,15,18]$. В частности, было рассмотрено взаимодействие кислорода с атомарными комплексами серебра и с кластером $\mathrm{Ag}_{5}$, закрепленным на полностью окисленной и содержащей кислородные вакансии поверхности $\mathrm{CeO}_{2}(111)[12,18]$.

В настоящей работе выполнено моделирование взаимодействия молекулы $\mathrm{O}_{2}$ с атомами, тримерами и тетрамерами серебра, закрепленными на поверхности наноструктурированного оксида $\mathrm{CeO}_{2}$, способного к непосредственному участию в окислительно-восстановительных превращениях [19]. В этом случае одним из главных факторов, определяющих свойства закрепленных на поверхности субнаноразмерных форм переходных металлов (ПМ), должна быть сила взаимодействия с подложкой $[3,6,20]$. В этой связи в сравнении с прочими технологически значимыми субстратными материалами оксид церия выделяется повышенной способностью к дисперсированию поверхностной фазы ПМ [6, 14, 21]. Другим важным фактором, определяющим размерное распределение закрепленных на поверхности $\mathrm{CeO}_{2}$ кластеров ПМ в условиях приготовления и функционирования катализаторов, должно быть взаимодействие этих кластеров с молекулой $\mathrm{O}_{2}$ [9], как, например, это может наблюдаться в случае частиц серебра на подложках $\mathrm{TiO}_{2}$ и $\mathrm{SiO}_{2}[22]$. Данное обстоятельство, в частности, подтверждается экспериментами, в которых наблюдалось образование оксидной $\mathrm{Ag}_{2} \mathrm{O}$ фазы, разделяющей фазы оксида $\mathrm{CeO}_{2}$ и закрепленного на его поверхности ультрадисперсного металлического серебра [1].

Ультрадисперсные формы серебра на поверхности оксида $\mathrm{CeO}_{2}$ часто характеризуются как лучшие катализаторы $[1,6,7,11]$. В таких катализаторах $[2,3,6]$ и в моделирующих их системах с хорошо определенными поверхностями $[4,18,21,23]$ в качестве доминирующей поверхностной фазы серебра были выявлены небольшие наночастицы. Также исследовалась поверхность $\mathrm{CeO}_{2}$ с изолированными ионами $\mathrm{Ag}^{+}[2,3,7]$. В экспериментах [11] было установлено, что при нанесении серебра на поверхность $\mathrm{CeO}_{2}$ увеличивается количество супероксидных групп. В ряде недавних исследований поверхность $\mathrm{CeO}_{2}$ рассматривалась в качестве подложки для атомов и кластеров серебра $[2,3,7,12,18,21,24,25,26]$ и других ПМ

$$
-283-
$$


[24-29]. В частности, были выполнены расчеты систем с атомом серебра на регулярных и дефектных по кислороду поверхностях $\mathrm{CeO}_{2}[12,18,24-26]$ и атомами серебра, замещающими ионы церия поверхности $\mathrm{CeO}_{2}(111)$ [30]. Не стало неожиданным, что обладающий хорошо развитой поверхностью оксид $\mathrm{CeO}_{2}$ в каталитических приложениях представлен его наноструктурированными разновидностями $[6,7,20]$. Использование в качестве подложек для ультрадисперсных частиц ПМ наноструктурированного $\mathrm{CeO}_{2}$ дает расширенный спектр возможностей появления выгодных для каталитических систем особенностей [7, 20, 31], так как поверхность таких подложек представлена разнотипными гранями и соединяющими их ребрами. При этом ожидается, что достаточно сильное взаимодействий данных структурных образований с атомами и частицами ПМ будет способствовать образованию поверхностных комплексов изолированных атомов и малых кластеров ПМ $[14,32,33]$. Также отмечалось, что образование легко восстанавливаемых кислородных группировок активных при окислении молекул $\mathrm{CH}_{4}$ становится более выгодным в присутствии поверхностных форм $\mathrm{Ag}$ на частицах $\mathrm{CeO}_{2}$ небольшого размера [6].

Взаимодействие атомов и небольших кластеров ПМ с наночастицами $\mathrm{CeO}_{2}$ исследовалось в нескольких недавних теоретических работах $[14,27,29,32]$. Однако исследования атомов и кластеров серебра, закрепленных на поверхности наночастиц $\mathrm{CeO}_{2}$, и их взаимодействия с молекулой $\mathrm{O}_{2}$ до сих пор не выполнялись. В настоящей работе мы рассматриваем локализацию адсорбционных комплексов атома, тримера и тетрамера серебра с $\mathrm{O}_{2}$ на поверхности модельной наночастицы $\mathrm{Ce}_{21} \mathrm{O}_{42}(\mathrm{NP})$ [34] как одной из недавно предложенных моделей для описания свойств наноструктурированного $\mathrm{CeO}_{2}[14,27,34-36]$. Целями настоящей работы являются: a) определение того, как свойства наночастиц $\mathrm{CeO}_{2}$ и закрепленных на них молекулярных форм серебра модифицируются вследствие их взаимного воздействия; б) получение количественных характеристик силы данного взаимодействия; с) характеризация адсорбции и возможной диссоциации молекулы $\mathrm{O}_{2}$ на молекулярных формах $\mathrm{Ag}$, закрепленных на поверхности частиц $\mathrm{CeO}_{2}$.

\section{Детали расчетов}

Расчеты методом функционала плотности (МФП) были выполнены с использованием пакета программ VASP [37, 38]. Неограниченные по спину собственные функции метода PAW (projector-augmented-waves) [39, 40] валентного пространства с одиннадцатью $\left(5 \mathrm{~s}^{1} 4 \mathrm{~d}^{10}\right)$ электронами от каждого атома $\mathrm{Ag}$, двенадцатью $\left(5 \mathrm{~s}^{2} 5 \mathrm{p}^{6} 6 \mathrm{~s}^{2} 5 \mathrm{~d}^{1} 4 \mathrm{f}^{1}\right)$ электронами от каждого атома Се и шестью $\left(2 \mathrm{~s}^{2} 2 \mathrm{p}^{4}\right)$ электронами от каждого атома О генерировались с помощью базиса плоских волн, ограниченных энергиями до 415 эВ. При этом применялись расчетная схема обобщенного градиентного приближения GGA+U [41] с параметризацией обменно-корреляционного функционала Пердью-Ванга PW91 [42] и добавлением поправок Хаббарда U, равных 4 эB, для 4f-состояний атома Се в соответствии с обоснованием предыдущих исследований [14, 27, 34, $36]$.

При моделировании наноструктурированного оксида $\mathrm{CeO}_{2}$ использовалась модельная наночастица $\mathrm{Ce}_{21} \mathrm{O}_{42}$, поверхность которой представлена гранями $\{100\}$ и $\{111\}$, а также соединяющими их реберными структурами (рис. 1). По данным экспериментов в [4], ультрадисперсные частицы серебра, главным образом, концентрируются вблизи ступенек, соединяющих поверх- 


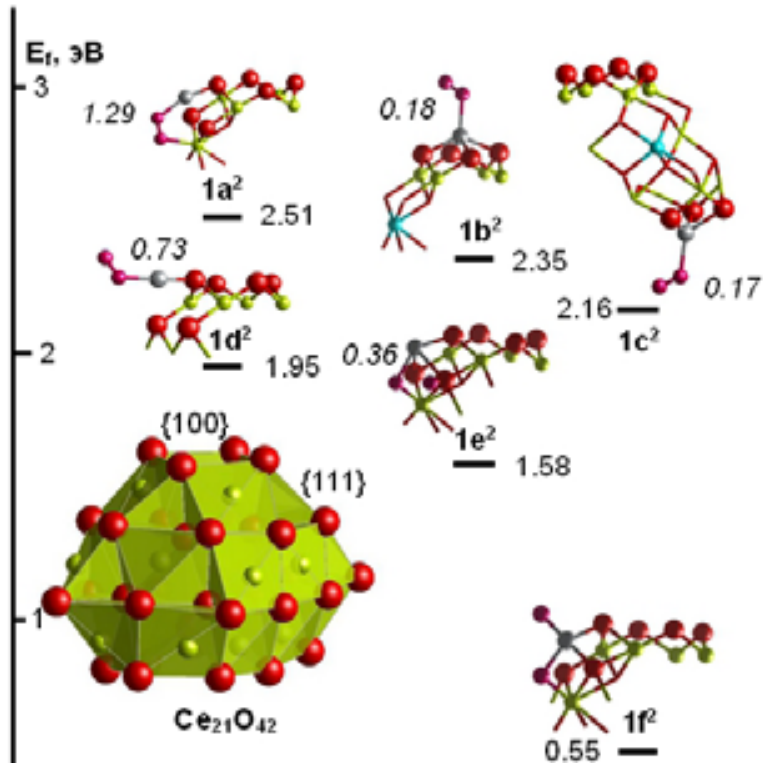

Рис. 1. Структура наночастицы $\mathrm{Ce}_{21} \mathrm{O}_{42}$ и комплексов молекулы $\mathrm{O}_{2}$ с атомом $\mathrm{Ag}$ на поверхности нанограней $\{100\}$ и $\{111\}$ этой частицы (для простоты для комплексов $\mathrm{O}_{2} \cdot \mathrm{Ag} / \mathrm{NP}$ показаны только атомы наночастицы вблизи фрагмента $\left.\mathrm{O}_{2} \cdot \mathrm{Ag}\right)$. Прямым и наклонным шрифтами даны энергии $\mathrm{E}_{\mathrm{f}}$ и $\mathrm{E}_{\mathrm{ad}}\left(\mathrm{O}_{2}\right)(\ni \mathrm{B})$ соответственно. Атомные сферы красного, серого, желто-зеленого и голубого цвета отвечают анионам $\mathrm{O}$, атомам и ионам $\mathrm{Ag}$, катионам $\mathrm{Ce}^{4+}$ и $\mathrm{Ce}^{3+}$ соответственно. Атомы О молекулы кислорода выделены розовым цветом и имеют меньший размер по сравнению с атомами О нанокластера.

ности разновысотных (111) террас. Реберные соединения NP являются аналогами данных ступенек. В связи с малым размером нанограней $\{100\}$ и $\{111\}$ атомы серебра и субнаноразмерные кластеры $\mathrm{Ag}_{\mathrm{n}}$ на данных поверхностях также находятся в непосредственной близости от соответствующих реберных соединений. В моделях для расчета каждой конечной системы, с решениями только для Г-точки обратного пространства, для исключения взаимодействий между $\mathrm{Ce}_{21} \mathrm{O}_{42}$ частицами в сопредельных периодических ячейках по каждой из трех декартовых координат использовались вакуумные промежутки в 1 нм.

В расчетах комплексов на поверхности $\mathrm{CeO}_{2}(111)$ рассматривались пластины из шести атомных слоев ячейки $\mathrm{Ce}_{18} \mathrm{O}_{36}$ с поверхностными векторами $\mathbf{a}$ и $\mathbf{b}$, фиксированными по соответствию решеточному параметру $\mathrm{a}_{0}$ в 0.54 нм [25] и межпластиночными расстояниями, равными 1.7 нм. При интегрировании в обратном пространстве применялась $3 \times 3 \times 1$ сетка $k$-точек Монкхорста-Пака [43]. При расчете барьеров диссоциации молекулы $\mathrm{O}_{2}$ путь минимальной энергии между МА- и ДА-изомерами описывался точками метода натянутой струны, и переходное состояние аппроксимировалось с использованием полиномиальных сплайнов (a string method scheme [44]). Частотный анализ переходных состояний не проводился, так как для каждого переходного состояния осуществляли построение реакционного пути и для каждой точки анализировали продольные и поперечные энергетические производные, что однозначно доказывало наличие на данном пути переходного состояния.

Энергия образования комплексов $\mathrm{O}_{2} \cdot \mathrm{Ag}_{\mathrm{n}} / \mathrm{Ce}_{21} \mathrm{O}_{42}$ и энергия адсорбции молекулы $\mathrm{O}_{2}$ рассчитывались по формулам (1) и (2) соответственно:

$$
-285-
$$




$$
\begin{aligned}
& \mathrm{E}_{\mathrm{f}}=\mathrm{E}\left(\mathrm{O}_{2}\right)+\mathrm{E}\left(\mathrm{Ag}_{\mathrm{n}}\right)+\mathrm{E}\left(\mathrm{Ce}_{21} \mathrm{O}_{42}\right)-\mathrm{E}\left(\mathrm{O}_{2} \cdot \mathrm{Ag}_{\mathrm{n}} / \mathrm{Ce}_{21} \mathrm{O}_{42}\right), \\
& \mathrm{E}_{\mathrm{ad}}\left(\mathrm{O}_{2}\right)=\mathrm{E}\left(\mathrm{O}_{2}\right)+\mathrm{E}\left(\mathrm{Ag}_{\mathrm{n}} / \mathrm{Ce}_{21} \mathrm{O}_{42}\right)-\mathrm{E}\left(\mathrm{O}_{2} \cdot \mathrm{Ag}_{\mathrm{n}} / \mathrm{Ce}_{21} \mathrm{O}_{42}\right) .
\end{aligned}
$$

\section{Результаты и обсуждение}

Согласно данным расчетов МФП при взаимодействии молекулы $\mathrm{O}_{2}$ с поверхностью стехиометрических моделей $\mathrm{CeO}_{2}$ значимо прочные адсорбционные комплексы не образуются $[12,36,45]$. Поэтому в настоящей работе мы рассматривали взаимодействие молекулы $\mathrm{O}_{2}$ или исключительно с атомами $\mathrm{Ag}$, или одновременно с атомами серебра и церия. Структуры низкоэнергетических изомеров таких комплексов, энергии их образования $\mathrm{E}_{\mathrm{f}}$ и энергии адсорбции молекулы кислорода $\mathrm{E}_{\mathrm{ad}}\left(\mathrm{O}_{2}\right)$ приводятся на рис. 1, 2 и 3. Различные изомеры комплексов промаркированы составными индексными обозначениями $\mathbf{n} \mathbf{L}^{\mathbf{x}}$, где $\mathbf{n}-$ число атомов серебра $(\mathrm{n}=1$, $3,4), \mathbf{L}$ - отвечающая алфавитному порядку позиция комплекса $\mathrm{O}_{2} \cdot \mathrm{Ag}_{\mathrm{n}} / \mathrm{NP}$ в ряду наиболее стабильных комплексов с одинаковым $\mathbf{n}$ (a - для самого стабильный комплекса, $\mathbf{b}-$ для второго по стабильности и т.д.), $\mathrm{x}$ - индекс $2 \mathrm{~S}+1$ спинового состояния ( $\mathrm{x}=1,2$ и 3 для синглета, дублета и триплета соответственно).

Взаимодействие с атомами серебра. Нами рассматривались три способа адсорбции молекулы $\mathrm{O}_{2}$ на адсорбентах $\mathrm{Ag} / \mathrm{NP}\{100\}$ и $\mathrm{Ag} / \mathrm{NP}\{111\}$ : молекулярная терминальная (с координацией $\mathrm{O}_{2}$ к атому $\mathrm{Ag}$ через один атом $\mathrm{O}$, см. $\mathrm{AgO}_{2}$-изомеры $\mathbf{1 b}^{2} \mathbf{1}^{\mathbf{2}}$ и $\mathbf{1 d}^{2}$, рис. 1), молекулярная мостиковая (с координацией $\mathrm{O}_{2}$ к атомам серебра и церия, см. $\mathrm{AgOO}\{\mathrm{Ce}\} / \mathrm{NP}\{111\}-$ $\mathbf{1 a}^{2}$ изомер, рис. 1) и диссоциативная (с распадом $\mathrm{O}_{2}$ на отдельные атомы, см. OAgO $\{\mathrm{Ce}\} /$ $\mathrm{NP}\{111\}$-изомеры $\left.\mathbf{1 e}^{2}, \mathbf{1 f}^{2}\right)$. Здесь и далее при обозначении типа адсорбционного комплекса символ Се в фигурных скобках указывает на расположение атома $\mathrm{O}$ или молекулы $\mathrm{O}_{2}$ в

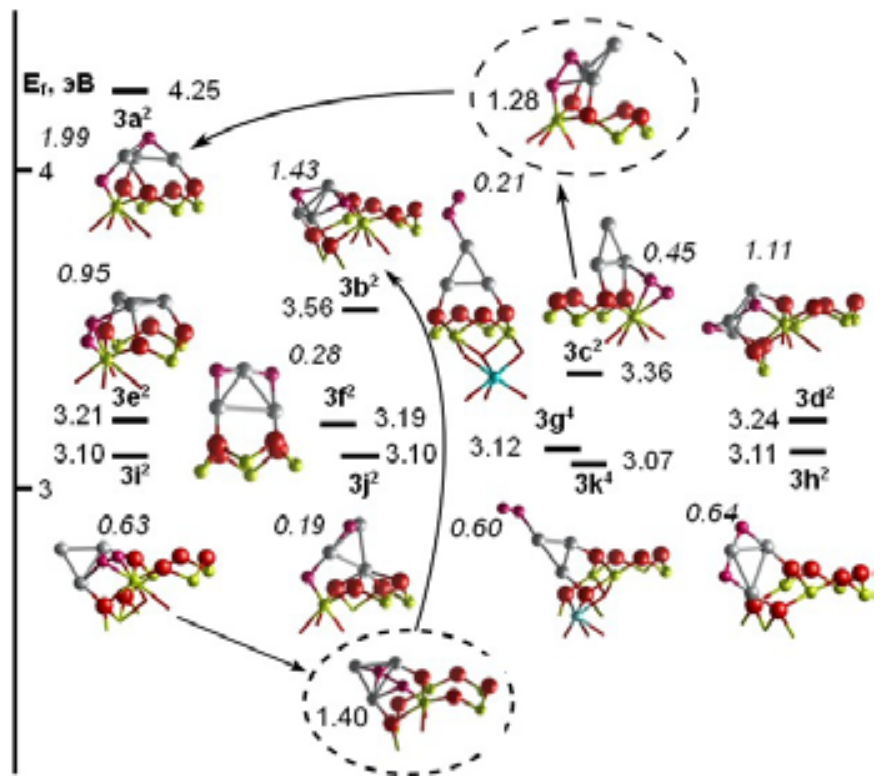

Рис. 2. Структура и энергии (в эВ) образования $\mathrm{E}_{\mathrm{f}}$ и адсорбции $\mathrm{E}_{\mathrm{ad}}\left(\mathrm{O}_{2}\right)$ комплексов $\mathrm{O}_{2}$ с кластером $\mathrm{Ag}_{3}$ на поверхности нанограней $\{100\}$ и $\{111\}$ частицы $\mathrm{Ce}_{21} \mathrm{O}_{42}$. Обозначения атомов см. в подписи к рис. 1. На эллиптических панелях приводятся энергии образования и структуры переходных состояний диссоциации адсорбированных молекул $\mathrm{O}_{2}$ 


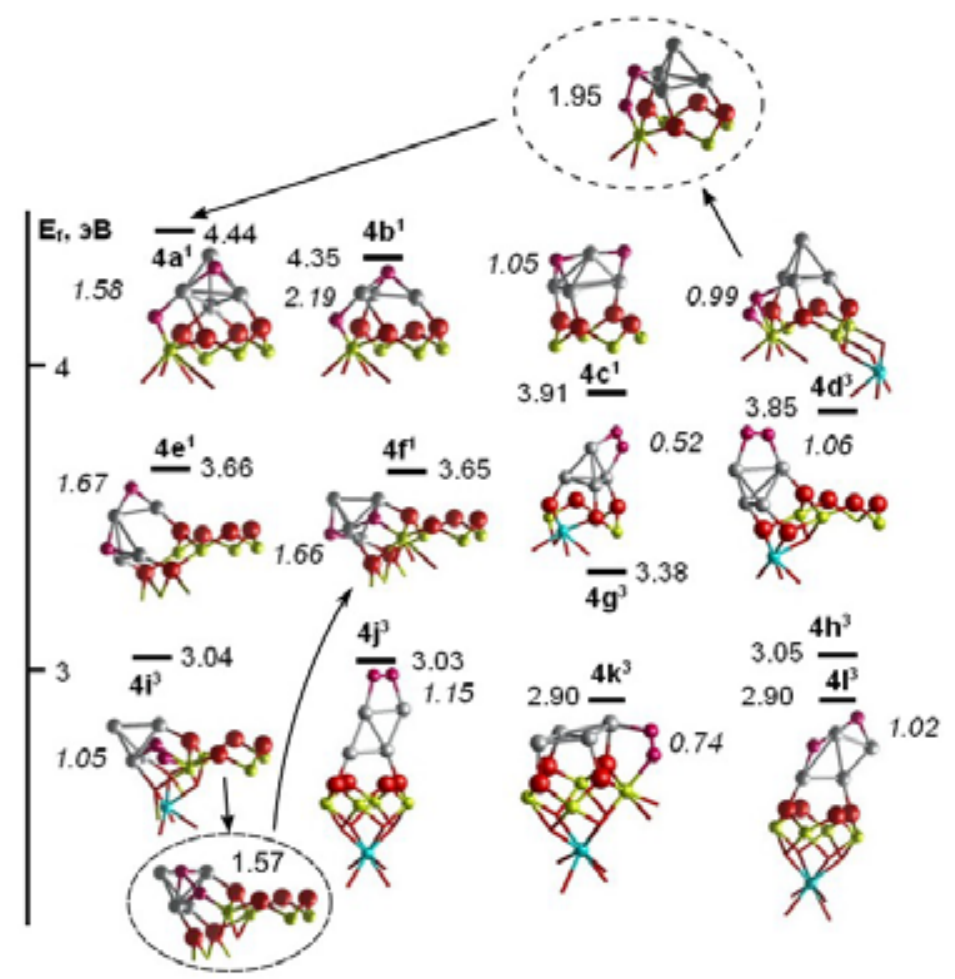

Рис. 3. Структура и энергии (в эВ) образования $\mathrm{E}_{\mathrm{f}}$ и адсорбции $\mathrm{E}_{\mathrm{ad}}\left(\mathrm{O}_{2}\right)$ комплексов $\mathrm{O}_{2}$ с кластером $\mathrm{Ag}_{4}$ на поверхности нанограней $\{100\}$ и $\{111\}$ частицы $\mathrm{Ce}_{21} \mathrm{O}_{42}$. Обозначения атомов см. в подписи к рис. 1. На эллиптических панелях приводятся энергии образования и структуры переходных состояний диссоциации адсорбированных молекул $\mathrm{O}_{2}$

мостиковой позиции между атомами серебра и одним из атомов церия наночастицы. Согласно расчетным данным молекула $\mathrm{O}_{2}$ не образует прочной связи с атомом серебра на наноповерхности $\{100\}$. Энергия $\mathrm{E}_{\text {ad }}\left(\mathrm{O}_{2}\right)$ для $\mathrm{AgO}_{2} / \mathrm{NP}\{100\}$-изомеров $\mathbf{1 b}^{2}$ и $\mathbf{1} \mathbf{c}^{2}$ составляет менее 0.2 эВ. Однако адсорбция $\mathrm{O}_{2}$ в терминальной моде на нанограни $\{111\}\left(\mathrm{AgO}_{2} / \mathrm{NP}\{111\}-\right.$ $\mathbf{1 d}^{2}$ изомер) рассчитана умеренно прочной, $\mathrm{E}_{\mathrm{ad}}\left(\mathrm{O}_{2}\right)=0.73$ эВ. Длины связи О-О в адсорбированной молекуле $\mathrm{O}_{2}$ для $\mathrm{AgO}_{2}$-изомеров, 127-130 пм, на 3-6 пм больше, чем в свободной молекуле $\mathrm{O}_{2}$. Расстояния $\mathrm{O}-\mathrm{Ag}$ (до атомов $\mathrm{O}_{2}$ ) составляют 233 пм. Энергия $\mathrm{E}_{\mathrm{ad}}\left(\mathrm{O}_{2}\right)$ в комплексе $1 \mathbf{d}^{2}$ сопоставима с энергиями 0.45 и 0.67 эВ, полученными в GGA-PW91+3 - [12] и GGA-PW91+4 - расчетах для аналогичного комплекса на поверхности $\mathrm{CeO}_{2}(111)$. Молекула $\mathrm{O}_{2}$ оказывается прочно связанной в мостиковой моде в комплексе $\mathrm{AgOO}\{\mathrm{Ce}\} / \mathrm{NP}\{111\}-\mathbf{1 a}^{2}$ c $\mathrm{E}_{\mathrm{ad}}\left(\mathrm{O}_{2}\right)=1.29$ эВ. Данный комплекс с $\mathrm{E}_{\mathrm{f}}$, достигающей 2.51 эВ, наиболее стабильный изомер среди комплексов $\mathrm{O}_{2}$ c $\mathrm{Ag} / \mathrm{NP}$. Расстояния O-Ag и O-Cе (до атомов $\mathrm{O}_{2}$ ) в изомере $\mathbf{1 a}^{2}$ составляют 223 и 245 пм соответственно. Длина связи О-О во фрагменте $\mathrm{O}_{2}$ в изомере $\mathbf{1 a}^{2}$ равняется 133 пм, что на 9 пм больше, чем в расчетах свободной молекулы. Из полученных данных следует, что при фиксации атомов серебра на поверхности наночастицы $\mathrm{Ce}_{21} \mathrm{O}_{42}$, главным образом на наногранях $\{111\}$ происходит значительное увеличение вероятности захвата приходящей молекулы $\mathrm{O}_{2}$. Кроме того, при образовании комплекса $\mathbf{1 a}^{2}$ наблюдается заметная активация адсорбированной молекулы $\mathrm{O}_{2}$. 
Мы рассмотрели возможность диссоциации молекулы $\mathrm{O}_{2}$ в наиболее стабильном комплекce 1a $\mathbf{a}^{2}$. Энергия $\mathrm{E}_{\mathrm{f}}$ полученного при диссоциации комплекса OAgO $\{\mathrm{Ce}\} / \mathrm{NP}\{111\}-\mathbf{1 \mathbf { f } ^ { 2 }}$ (рис. 1) с атомами О в мостиковых $\mathrm{Ag}-\mathrm{O}-\mathrm{Ce}$ и $\mathrm{Ag}-\mathrm{O}-\mathrm{Ag}$ положениях оказывается на $\sim 2$ эВ меньше, чем для изомера $1 \mathbf{a}^{2}$. Конверсия изомера $1 \mathbf{f}^{2}$ в $1 \mathbf{a}^{2}$ требует преодоления барьера в $\sim 0.4$ эВ. В другой равновесной ДА-структуре, комплексе $1 \mathrm{e}^{2}$, один из атомов О находится в мостиковом Ag-O-Cе положении, а второй - связан с атомом О решетки NP с образованием новой ОО-группировки. Структура $1 \mathbf{e}^{2}$ менее стабильна, чем изомер $1 \mathbf{a}^{2}$ на 0.93 эВ. Конверсия структуры $1 \mathbf{e}^{2}$ в $1 \mathbf{a}^{2}$ требует энергии активации 0.84 эВ. Таким образом, ОО группировка в $\mathrm{AgOO}\{\mathrm{Ce}\}$-комплексе $\mathbf{1 a}^{2}$ оказывается доминирующей активной формой поверхностного кислорода, полученного адсорбцией молекулы $\mathrm{O}_{2}$ на адсорбенте $\mathrm{Ag} / \mathrm{NP}$. Подобные структуры образуются также и при адсорбции молекулы $\mathrm{O}_{2}$ на тримерах и тетрамерах серебра, закрепленных на $\mathrm{Ce}_{21} \mathrm{O}_{42}$ (см. ниже).

Взаимодействие с тримерами и тетрамерами серебра. Нами определены равновесные структуры комплексов $\mathrm{O}_{2} \cdot \mathrm{Ag}_{3} / \mathrm{NP}$ (рис. 2) и $\mathrm{O}_{2} \cdot \mathrm{Ag}_{4} / \mathrm{NP}$ (рис. 3) с молекулой $\mathrm{O}_{2}$, адсорбированной в терминальной моде $\left(\mathrm{Ag}_{3} \mathrm{O}_{2}\right.$-изомеры $\left.\mathbf{3} \mathbf{g}^{4}, \mathbf{3} \mathbf{k}^{4}\right)$ или в мостиковой моде с координацией через оба атома О ( $\mathrm{Ag}_{4} \mathrm{OO}$-изомеры $\mathbf{4 g}^{\mathbf{3}}, \mathbf{4} \mathbf{h}^{\mathbf{3}}$ и $\mathbf{4} \mathbf{j}^{3} ; \mathrm{Ag}_{\mathrm{n}} \mathrm{OO}\{\mathrm{Ce}\}$-изомеры $\mathbf{3} \mathbf{c}^{2}, \mathbf{3 e}^{\mathbf{2}}, \mathbf{3 i}^{\mathbf{2}}, \mathbf{4 d}^{\mathbf{3}}, \mathbf{4 i}^{\mathbf{3}}$ и $\left.\mathbf{4} \mathbf{k}^{\mathbf{3}}\right)$. Также определены равновесные структуры, полученные ДА-адсорбцией молекулы $\mathrm{O}_{2}$ c $\mathrm{Ag}-\mathrm{O}-\mathrm{Ag}$

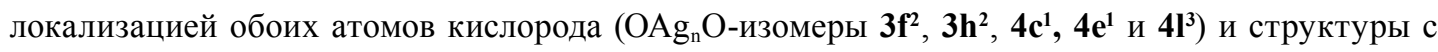

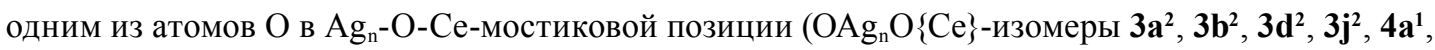
$4 b^{1}$ и $\left.4 f^{1}\right)$.

$\mathrm{B} \mathrm{Ag}_{3} \mathrm{O}_{2}$-комплексах длина О-О связи лишь на 2 пм больше, чем в свободной молекуле $\mathrm{O}_{2}$. Более значительные инкременты длин О-О связи в 6-10 пм найдены для $\mathrm{Ag}_{4} \mathrm{OO}-, \mathrm{Ag}_{3} \mathrm{OO}\{\mathrm{Ce}\}-$ и $\mathrm{Ag}_{4} \mathrm{OO}\{\mathrm{Ce}\}$-изомеров. Кратчайшие расстояния $\mathrm{O}-\mathrm{Ag}$ для $\mathrm{Ag}_{\mathrm{n}} \mathrm{OO}\{\mathrm{Ce}\}$-изомеров достигают довольно больших величин, 272-298 пм $(\mathrm{n}=3)$ и 245-255 пм $(\mathrm{n}=4)$. Для остальных комплексов расстояния O-Ag составляют 223-238 пм. Более короткие связи O-Ag были получены для ДАкомплексов, 199-234 пм. Кратчайшие расстояния О-Се в ДА-комплексах рассчитаны в интервале 198-210 пм.

Согласно полученным данным молекулярная адсорбция $\mathrm{O}_{2}$ на $\mathrm{Ag}_{3} / \mathrm{NP}\{100\}$ приводит к образованию слабосвязанного $\mathrm{Ag}_{3} \mathrm{O}_{2}$-изомера $3 \mathbf{g}^{4} \mathrm{c} \mathrm{E}_{\mathrm{ad}}\left(\mathrm{O}_{2}\right)=0.21$ эВ. Такой же небольшой величиной $\mathrm{E}_{\mathrm{ad}}\left(\mathrm{O}_{2}\right)$ характеризуется и газофазный адсорбционный комплекс $\mathrm{Ag}_{3} \mathrm{O}_{2}^{+}[15]$. Умеренно большая энергия $\mathrm{E}_{\mathrm{ad}}\left(\mathrm{O}_{2}\right)=0.60$ эВ рассчитана для однотипного с $\mathbf{3} \mathbf{g}^{4}$ изомера на поверхности нанограни $\{111\}$, комплекса $3 \mathbf{k}^{4}$. Также умеренно большие значения $\mathrm{E}_{\mathrm{ad}}\left(\mathrm{O}_{2}\right), 0.45$ эВ $\left(\mathbf{3} \mathbf{c}^{2}\right)$ и 0.63 эВ $\left(\mathbf{3 i}^{2}\right)$ были рассчитаны для $\mathrm{Ag}_{3} \mathrm{OO}\{\mathrm{Ce}\}$-изомеров с кластером $\mathrm{Ag}_{3}$, стоящим перпендикулярно на $\{100\}$ и $\{111\}$ наногранях соответственно. Заметно более сильно связанной с $\mathrm{E}_{\mathrm{ad}}\left(\mathrm{O}_{2}\right)=0.95$ эВ оказывается молекула $\mathrm{O}_{2}$ в $\mathrm{Ag}_{3} \mathrm{OO}\{\mathrm{Ce}\}$-комплексе $3 \mathrm{e}^{2}$ с лежащим параллельно поверхности кластером $\mathrm{Ag}_{3}$. В целом адсорбция $\mathrm{O}_{2}$ на $\mathrm{Ag}_{3}$-кластерах, закрепленных на поверхности $\{111\}$ нанограней, приводит к образованию более прочно связанных комплексов в сравнении с аналогичными комплексами на поверхности нанограней $\{100\}$. Энергии $\mathrm{E}_{\mathrm{f}}$ наиболее стабильных МА-комплексов $\mathrm{O}_{2} \cdot \mathrm{Ag}_{3} / \mathrm{NP}\{100\}$ и $\mathrm{O}_{2} \cdot \mathrm{Ag}_{3} / \mathrm{NP}\{111\}$ составляют 3.36 и 3.10 эВ соответственно. Представляет интерес сопоставление связывания молекулы $\mathrm{O}_{2}$ на $\mathrm{Ag}_{3} / \mathrm{NP}\{111\}$ и на поверхностных комплексах $\mathrm{Ag}_{3} / \mathrm{CeO}_{2}(111)$. Энергии адсорбции $\mathrm{E}_{\text {ad }}\left(\mathrm{O}_{2}\right)$ для структур $\mathrm{Ag}_{3} \mathrm{O}_{2} / \mathrm{CeO}_{2}(111)$ и $\mathrm{Ag}_{3} \mathrm{OO} / \mathrm{CeO}_{2}(111)$ были рассчитаны равными 0.15 и 0.20 эВ соответственно, что близко к энергиям $\mathrm{E}_{\mathrm{ad}}\left(\mathrm{O}_{2}\right)$ на кластерах $\mathrm{Ag}_{3}$, координированных на наногранях $\{100\}$ частицы $\mathrm{Ce}_{21} \mathrm{O}_{42}$. Та- 
ким образом, связывание молекулы $\mathrm{O}_{2}$ на тримерах серебра критическим образом зависит от морфологии подложки оксида церия и оказывается заметно более сильным для комплексов на наногранях $\{111\}$ частицы $\mathrm{Ce}_{21} \mathrm{O}_{42}$.

Молекулярная адсорбция $\mathrm{O}_{2}$ на тетрамерах серебра, приводящая к образованию $\mathrm{Ag}_{4} \mathrm{OO}$ и $\mathrm{Ag}_{4} \mathrm{OO}\{\mathrm{Ce}\}$-структур, характеризуется значительными адсорбционными энергиями $\mathrm{E}_{\mathrm{ad}}\left(\mathrm{O}_{2}\right)$ : 0.52 эВ для $\mathrm{Ag}_{4} \mathrm{OO} / \mathrm{NP}\{100\}-\mathbf{4 g}^{3}, 0.74$ эВ для $\mathrm{Ag}_{4} \mathrm{OO}\{\mathrm{Ce}\} / \mathrm{NP}\{100\}-4 \mathbf{k}^{3}, 0.99$ эВ для $\mathrm{Ag}_{4} \mathrm{OO}\{\mathrm{Ce}\} /$ $\mathrm{NP}\{100\}-\mathbf{4 d}^{3}, 1.05$ эВ для $\mathrm{Ag}_{4} \mathrm{OO}\{\mathrm{Ce}\} / \mathrm{NP}\{111\}-4 \mathbf{i}^{3}, 1.06$ эВ для $\mathrm{Ag}_{4} \mathrm{OO} / \mathrm{NP}\{111\}-\mathbf{4 h} \mathbf{h}^{3}, 1.15$ эВ для $\mathrm{Ag}_{4} \mathrm{OO} / \mathrm{NP}\{100\}-\mathbf{4} \mathbf{j}^{3}$ изомеров. Комплексы $\mathbf{4} \mathbf{k}^{3}$ и $\mathbf{4} \mathbf{j}^{3}$ содержат ромбический кластер $\mathrm{Ag}_{4}$ В остальных МА-комплексах кластер $\mathrm{Ag}_{4}$ имеет тетраэдрическую структуру. Энергии Е $\mathrm{E}_{\mathrm{f}}$ наиболее стабильных МА-комплексов $\mathrm{O}_{2} \cdot \mathrm{Ag}_{4} / \mathrm{NP}\{100\}$ и $\mathrm{O}_{2} \cdot \mathrm{Ag}_{4} / \mathrm{NP}\{111\}$ равны 3.85 и 3.05 эВ соответственно. Энергии $\mathrm{E}_{\text {ad }}\left(\mathrm{O}_{2}\right)$ в наиболее стабильных комплексах $\mathrm{O}_{2} \cdot \mathrm{Ag}_{4} / \mathrm{NP}\{100\}$ и $\mathrm{O}_{2} \cdot \mathrm{Ag}_{4} / \mathrm{NP}\{111\}, 0.99$ и 1.06 эВ соответственно рассчитаны значительно большими, чем в аналогичных энергетически предпочтительных комплексах тримера серебра (0.45 и 0.63 эВ). Аналогично структурам с тримерами серебра молекулярный $\mathrm{O}_{2}$ в комплексах тетрамеров $\mathrm{O}_{2} \cdot \mathrm{Ag}_{4} / \mathrm{Ce}_{21} \mathrm{O}_{42}$ связан более сильно на нанограни $\{111\}$, чем на поверхности $\mathrm{CeO}_{2}(111), \mathrm{E}_{\mathrm{b}}\left(\mathrm{O}_{2}\right)=0.77$ эВ. Величины $\mathrm{E}_{\mathrm{ad}}\left(\mathrm{O}_{2}\right)$ для MA-комплексов $\mathrm{O}_{2}$ на газофазных кластерах $\mathrm{Ag}_{3}$ и $\mathrm{Ag}_{4}$, рассчитанные в GGA-приближении, варьируются в интервалах 0.9-1.2 и 0.3-0.5 эВ соответственно [13, 16, 17]. В сравнении с комплексами свободных кластеров $\mathrm{Ag}_{\mathrm{n}}$ комплексы $\mathrm{Ag}_{3} \mathrm{OO}\{\mathrm{Ce}\} / \mathrm{NP}$ оказываются связанными менее прочно, $\mathrm{E}_{\mathrm{ad}}\left(\mathrm{O}_{2}\right)=0.45-0.95$ эВ, а комплексы $\mathrm{Ag}_{4} \mathrm{OO}\{\mathrm{Ce}\} / \mathrm{NP}$ более прочно, $\mathrm{E}_{\mathrm{ad}}\left(\mathrm{O}_{2}\right), 0.74-1.05$ эB. Такое изменение относительных энергий связывания молекулы $\mathrm{O}_{2}$ на кластерах $\mathrm{Ag}_{3}$ и $\mathrm{Ag}_{4}$ при переходе от свободных к закрепленным на поверхности частицам соответствует ожидаемому эффекту их частичного окисления. Однако связывание молекулярного $\mathrm{O}_{2}$ с кластерами $\mathrm{Ag}_{3}$ и $\mathrm{Ag}_{4}$, закрепленными на наночастице, оказывается заметно более сильным, чем $\sim 0.2$ и $\sim 0.5$ эВ для свободных комплексов $\left[\mathrm{Ag}_{3} \mathrm{O}_{2}\right]^{+}$и $\left[\mathrm{Ag}_{4} \mathrm{OO}\right]^{+}$соответственно $[15,16]$.

Ранее в МФП-расчетах [15] моделировалась молекулярная и диссоциативная адсорбция молекулы $\mathrm{O}_{2}$ на кластерах $\mathrm{Ag}_{3}$ и $\mathrm{Ag}_{4}$, привитых к гидроксилированной поверхности $\mathrm{SiO}_{2}$ замещением $\mathrm{H}$ атомов силанольных групп $\left(\mathrm{Ag}_{\mathrm{n}}\right.$, координированный к немостиковым кислородным центрам · $\mathrm{O}-\mathrm{Si} \equiv$, NonBridging Oxygen - NBO). Кластеры $\mathrm{Ag}_{\mathrm{n}}$ в данных системах, как и в комплексах $\mathrm{Ag}_{\mathrm{n}} / \mathrm{NP}$, находятся в электронно-дефицитном состоянии. Связывание молекулы $\mathrm{O}_{2}$ в комплексах $\mathrm{Ag}_{3} / \mathrm{NP}$ определенно является более сильным, чем на $\mathrm{Ag}_{3} / \mathrm{SiO}_{2}(\mathrm{NBO})$ - адсорбентах, для которых величина $\mathrm{E}_{\mathrm{ad}}\left(\mathrm{O}_{2}\right)$ составила менее 0.1 эВ [15]. Напротив, величины $\mathrm{E}_{\mathrm{ad}}\left(\mathrm{O}_{2}\right)$ для тетрамеров серебра сопоставимы: 1.01 эВ для комплекса $\mathrm{Ag}_{4} \mathrm{OO} / \mathrm{SiO}_{2}(\mathrm{NBO})$ [15] и 1.06 эВ - для изомера $\mathrm{Ag}_{4} \mathrm{OO} / \mathrm{NP}\{111\}-\mathbf{4 h} \mathbf{h}^{3}$.

Значительные энергии адсорбции $\mathrm{E}_{\text {ad }}\left(\mathrm{O}_{2}\right)$ (0.45-1.29 эВ), увеличенные на 10 пм длины О-О связи и близкие к единице магнитные моменты адсорбированных молекул $\mathrm{O}_{2}(0.82-0.86 \mu \mathrm{B})$ в структурах $\mathrm{AgOO}\{\mathrm{Ce}\}, \mathrm{Ag}_{3} \mathrm{OO}\{\mathrm{Ce}\}$ и $\mathrm{Ag}_{4} \mathrm{OO}\{\mathrm{Ce}\}$ с мостиковой координацией молекулы кислорода, свидетельствуют о том, что $\mathrm{O}_{2}$ в данных комплексах стабилизируются в форме супероксидных групп $\mathrm{O}_{2}^{-}[15,36,45]$. В частности, похожие характеристики были недавно рассчитаны для супероксидных групп, образующихся на содержащих $\mathrm{Ce}^{3+}$-ионы наночастицах оксида церия [36]. Повышенная стабильность $\mathrm{Ag}_{\mathrm{n}} \mathrm{OO}\{\mathrm{Ce}\}$-комплексов согласуется с установленной в экспериментах повышенной генерацией супероксидных групп при нанесении серебра на поверхность наноструктурированного оксида $\mathrm{CeO}_{2}[11]$. 
Диссоциативная адсорбция кислорода. В предыдущих МПФ-расчетах (с ВР- и РВЕфункционалами) моделировалась диссоциативная адсорбция молекулы $\mathrm{O}_{2}$ на газофазных $\mathrm{Ag}_{\mathrm{n}}$, $\mathrm{Ag}_{\mathrm{n}}{ }^{+}$и на $\mathrm{Ag}_{\mathrm{n}} / \mathrm{SiO}_{2}(\mathrm{NBO})$ адсорбентах с $\mathrm{n}=3-4[13,15]$. Значения $\mathrm{E}_{\mathrm{ad}}\left(\mathrm{O}_{2}\right)$ для ДА-комплексов свободных $\mathrm{Ag}_{3}, \mathrm{Ag}_{4}$ и для $\mathrm{Ag}_{4} / \mathrm{SiO}_{2}(\mathrm{NBO})$ были рассчитаны равными $0.4, \sim 0.2$ и $\sim 1.2$ эВ [13, 15] соответственно. Образование ДА-комплексов с катионами $\mathrm{Ag}_{3}{ }^{+}, \mathrm{Ag}_{4}{ }^{+}$и с $\mathrm{Ag}_{3} / \mathrm{SiO}_{2}(\mathrm{NBO})-$ адсорбентом оказывается термодинамически невыгодным [13, 15]. В настоящей работе энергия $\mathrm{E}_{\mathrm{ad}}\left(\mathrm{O}_{2}\right)$ для ДА-комплекса $\mathrm{OAg}_{3} \mathrm{O} / \mathrm{NP}\{100\}-\mathbf{3 f}^{2}$ была рассчитана равной 0.28 эВ, что немного меньше, чем для ДА-комплекса свободного кластера $\mathrm{Ag}_{3}$, и немного больше, чем для МА-комплекса $\mathrm{Ag}_{3} \mathrm{O}_{2} / \mathrm{NP}\{100\}-\mathbf{3 g}^{4}$ (0.21 эВ). Значительно более сильное связывание молекулы $\mathrm{O}_{2}\left(\mathrm{E}_{\mathrm{ad}}\left(\mathrm{O}_{2}\right)=0.64\right.$ эB) было найдено для комплекса $\mathrm{OAg}_{3} \mathrm{O} / \mathrm{NP}\{111\}-3 \mathbf{h}^{2}$. Данное значение близко к энергии $\mathrm{E}_{\text {ad }}\left(\mathrm{O}_{2}\right)$ МА-изомеров $\mathrm{Ag}_{3} \mathrm{O}_{2} / \mathrm{NP}\{111\}-3 \mathbf{k}^{4}$ (0.60 эВ) и $\mathrm{Ag}_{3} \mathrm{OO}\{\mathrm{Ce}\} / \mathrm{NP}\{111\}-3 \mathbf{i}^{2}$ (0.63 эВ). Низкоэнергетические МА-комплексы на $\mathrm{Ag}_{3} / \mathrm{NP}\{100\}$ и $\mathrm{Ag}_{3} / \mathrm{NP}\{111\}$ адсорбентах, а также ДА-комплексы $\mathrm{OAg}_{3} \mathrm{O} / \mathrm{NP}\{100\}$ и $\mathrm{OAg}_{3} \mathrm{O} / \mathrm{NP}\{111\}$ имеют близкие энергии $\mathrm{E}_{\mathrm{f}}, 3.07-3.36$ эВ. ДА-изомеры $\mathrm{OAg}_{3} \mathrm{O}\{\mathrm{Ce}\} / \mathrm{NP}\{111\}-\mathbf{3 b}^{2}$ и $\mathrm{OAg}_{3} \mathrm{O}\{\mathrm{Ce}\} / \mathrm{NP}\{100\}-\mathbf{3 a}^{2}$ с ориентацией плоскости $\mathrm{Ag}_{3}$ треугольника параллельно поверхности грани характеризуются весьма большими энергиями $\mathrm{E}_{\mathrm{ad}}\left(\mathrm{O}_{2}\right)$, равными 1.43 и 1.99 эВ соответственно. Последний из этих изомеров с $\mathrm{E}_{\mathrm{f}}=4.25$ эВ оказывается наиболее стабильным комплексом молекулы $\mathrm{O}_{2} \mathrm{c} \mathrm{Ag} / \mathrm{NP}$. Таким образом, как молекулярная, так и диссоциативная адсорбция $\mathrm{O}_{2}$ на $\mathrm{Ag}_{3}$, закрепленном на наногранях $\{100\}$ и $\{111\}$, приводит к образованию наиболее стабильных изомеров в тех случаях, когда формируются $\mathrm{Ag}_{3} \mathrm{OO}\{\mathrm{Ce}\}-$ и $\mathrm{OAg}_{3} \mathrm{O}\{\mathrm{Ce}\}$-структуры, в которых атом кислорода адсорбата $\mathrm{O}_{2}$ находится в контакте с атомами церия подложки.

Низкоэнергетические $\mathrm{OAg}_{4} \mathrm{O}$-комплексы на наногранях $\{100\}$ и $\{111\}$ представлены соответственно $\mathbf{4} \mathbf{c}^{1}$ - и $\mathbf{4} \mathbf{e}^{1}$-изомерами с тетраэдрическим $\mathrm{Ag}_{4}$ и $\mathrm{E}_{\text {ad }}\left(\mathrm{O}_{2}\right)$, составляющими 1.05 и 1.67 эВ, а также изомером $4 \mathbf{1}^{3}$ с ромбическим $\mathrm{Ag}_{4}$ на нанограни $\{100\}$ с $\mathrm{E}_{\mathrm{ad}}\left(\mathrm{O}_{2}\right)$, равной $1.02{ } \mathrm{~B}$. $\mathrm{E}_{\mathrm{ad}}\left(\mathrm{O}_{2}\right)$ для $\mathbf{4} \mathbf{e}^{1}$ больше, чем для $\mathbf{4} \mathbf{c}^{1}$, однако адсорбент $\mathrm{Ag}_{4} / \mathrm{NP}\{100\}$ более устойчив, чем $\mathrm{Ag}_{4} / \mathrm{NP}\{111\}$, и в итоге $4 \mathbf{c}^{1}$-комплекс оказывается на 0.25 эВ более стабильным. Комплекс $4 \mathbf{c}^{1}$ также на 0.06 эВ более стабилен, чем наиболее стабильный $\mathrm{O}_{2} \cdot \mathrm{Ag}_{4} / \mathrm{NP}$ MA-комплекс. Величина $\mathrm{E}_{\mathrm{ad}}\left(\mathrm{O}_{2}\right)$ для $\mathbf{4} \mathbf{c}^{1}$ и $\mathbf{4 \mathbf { I } ^ { 3 }}$ оказывается немного меньшей, чем для комплекса $\mathrm{OAg}_{4} \mathrm{O} / \mathrm{SiO}_{2}(\mathrm{NBO})$ [15]. $\mathrm{OAg}_{4} \mathrm{O}\{\mathrm{Ce}\}$ комплексы на грани $\{100\}$ представлены двумя изомерами: с фрагментом $\mathrm{Ag}_{4}$ в виде тетраэдра (структура $\mathbf{4} \mathbf{a}^{1}$ ) и ромба (структура $\left.\mathbf{4} \mathbf{b}^{1}\right)$, а также изомером $\mathbf{4} \mathbf{f}^{1}$ с тетраэдрическим фрагментом $\mathrm{Ag}_{4}$ на NP\{111\}. Изомеры с расположенными на поверхности $\mathrm{NP}\{100\} \mathrm{OAg}_{4} \mathrm{O}\{\mathrm{Ce}\}$-фрагментами с энергиями $\mathrm{E}_{\mathrm{f}}$, равными 4.44 и 4.35 эВ, оказываются стабильнее более чем на 0.7 эВ, чем ком-

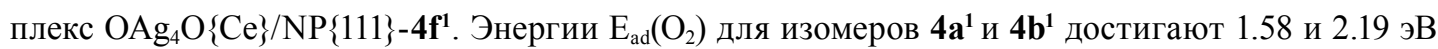
соответственно. В целом полученные результаты по адсорбции кислорода на адсорбентах $\mathrm{Ag}_{3} /$ $\mathrm{NP}$ и $\mathrm{Ag}_{4} / \mathrm{NP}$ указывают на предпочтительность ДА-комплексов с Ag-O-Се-связями.

Образование стабильных $\mathrm{Ag}_{\mathrm{n}} \mathrm{OO}\{\mathrm{Ce}\}$-групп на мономерах, тримерах и тетрамерах серебра, локализованных на наногранях $\{100\}$ и $\{111\}$, и даже более стабильных $\mathrm{OAg}_{\mathrm{n}} \mathrm{O}\{\mathrm{Ce}\}$-структур находится в соответствии с данными экспериментов [6], указывающих на то, что присутствие поверхностных форм серебра на наноструктурированном оксиде $\mathrm{CeO}_{2}$ приводит к образованию легко восстанавливаемых форм кислорода.

Ранее в расчетах МФП [13] было установлено, что диссоциативная адсорбция $\mathrm{O}_{2}$ на свободных частицах $\mathrm{Ag}_{\mathrm{n}}$ начинает преобладать над молекулярной адсорбцией при $\mathrm{n}>5$. 
Однако диссоциативная адсорбция была рассчитана предпочтительной уже для $\mathrm{Ag}_{4}$, привитого к поверхности оксида $\mathrm{SiO}_{2}[15]$. По данным наших расчетов, низкоэнергетические ДА-изомеры оказываются более стабильными, чем низкоэнергетические МА-изомеры на $\sim 0.45$ эВ для адсорбента $\mathrm{Ag}_{3} / \mathrm{NP}\{111\}$, на $\sim 0.6$ эВ для адсорбентов $\mathrm{Ag}_{4} / \mathrm{NP}\{100\}$ и $\mathrm{Ag}_{4} / \mathrm{NP}\{111\}$ и на $\sim 0.9$ эВ для $\mathrm{Ag}_{3} / \mathrm{NP}\{100\}$. Ранее в нашей работе [15] были выполнены расчеты энергии активации для диссоциации $\mathrm{O}_{2}$ в $\mathrm{Ag}_{3} \mathrm{OO}-$ и $\mathrm{Ag}_{4} \mathrm{OO}$-комплексах на поверхности $\mathrm{SiO}_{2}$. В настоящей работе рассчитаны барьеры диссоциации для низкоэнергетических $\mathrm{Ag}_{3} \mathrm{OO}\{\mathrm{Ce}\}-$ и $\mathrm{Ag}_{4} \mathrm{OO}\{\mathrm{Ce}\}$-комплексов (структуры и энергии переходных состояний приведены на рис. 2 и 3). Полученные барьеры, 1.47-2.07 эВ, оказываются заметно меньшими, чем барьеры для диссоциации $\mathrm{O}_{2}$, координированной к $\mathrm{Ag}_{3} / \mathrm{SiO}_{2}(\mathrm{NBO})$ и $\mathrm{Ag}_{4} / \mathrm{SiO}_{2}(\mathrm{NBO})$ с тетраэдрическим $\mathrm{Ag}_{4}, 3.28$ и 2.67 эВ [15] соответственно. Тем не менее полученные для $\mathrm{Ag}_{\mathrm{n}} \mathrm{OO}\{\mathrm{Ce}\}$ комплексов барьеры диссоциации свидетельствуют о низких скоростях реакций окисления, обусловленных диссоциацией $\mathrm{O}_{2}$. В условиях каталитического процесса, когда реакционные промоторы подвержены многократным окислительно-восстановительным преобразованиям, ожидаемые скорости реакций диссоциации $\mathrm{O}_{2}$ должны быть слишком небольшими для обеспечения значимых концентраций продуктов диссоциативной адсорбции. Поэтому следует ожидать, что супероксидные группы будут оказываться доминирующей формой активированного кислорода для каждого из рассмотренных субстратов $\mathrm{Ag}_{\mathrm{n}} / \mathrm{NP}$. Данное предположение, справедливое для предельно дисперсированных форм серебра, соответствует экспериментальным данным о быстром превращении $\mathrm{Ag}_{2} \mathrm{O}$ покрытий на поверхности оксида $\mathrm{CeO}_{2}$ в элементы фазы металлического серебра в ходе реакций каталитического дожигания сажи [3] и выводам [11] о том, что использование оксида $\mathrm{CeO}_{2}$ с закрепленными на поверхности наноструктурированными частицами серебра приводит к повышению каталитической активности в реакциях окисления в связи с усиленным образованием супероксидных групп.

Как уже отмечалось, в экспериментах с катализаторами $\mathrm{Ag} / \mathrm{CeO}_{2}$ и в моделирующих их системах с хорошо определенной структурой поверхности поверхностные формы серебра были представлены комплексами изолированных атомов серебра $[2,3,7]$ или же металлическими частицами, содержащими сотни агломерированных атомов $[2-4,6,18,21,23]$. Однако поверхностные комплексы кластеров серебра, содержащие только несколько атомов, также предположительно могут существовать в концентрации, зависящей от их относительной стабильности в сравнении с атомарными комплексами серебра. Согласно расчетным данным стабильность низкоэнергетических $\mathrm{O}_{2}$-комплексов с $\mathrm{Ag}_{\mathrm{n}} / \mathrm{NP}\{100\}$ и $\mathrm{Ag}_{\mathrm{n}} / \mathrm{NP}\{111\}$ понижается в ряду $\mathrm{AgOO}\{\mathrm{Ce}\} /$ $\mathrm{NP}\{111\}>\mathrm{AgO}_{2} / \mathrm{NP}\{100\}>\mathrm{OAg}_{3} \mathrm{O}\{\mathrm{Ce}\} / \mathrm{NP}\{100\}>\mathrm{OAg}_{4} \mathrm{O}\{\mathrm{Ce}\} / \mathrm{NP}\{100\}>\mathrm{OAg}_{3} \mathrm{O}\{\mathrm{Ce}\} / \mathrm{NP}\{111\}>$ $\mathrm{Ag}_{3} \mathrm{OO}\{\mathrm{Ce}\} / \mathrm{NP}\{100\}>\mathrm{Ag}_{4} \mathrm{OO}\{\mathrm{Ce}\} / \mathrm{NP}\{100\}>\mathrm{OAg}_{4} \mathrm{O} / \mathrm{NP}\{111\}>\mathrm{Ag}_{3} \mathrm{OO}\{\mathrm{Ce}\} / \mathrm{NP}\{111\}>\mathrm{Ag}_{4} \mathrm{OO} /$ $\mathrm{NP}\{111\}$. МА-комплексы $\mathrm{O}_{2}$, как на Ag/NP\{111\}, так и на Ag/NP\{100\} - адсорбентах оказываются более стабильными, чем любой из $\mathrm{O}_{2}$ адсорбционных комплексов на $\mathrm{Ag}_{3} / \mathrm{NP}-$ и $\mathrm{Ag}_{4} / \mathrm{NP}-$ адсорбентах. Образование наиболее низкоэнергетического адсорбционного комплекса $\mathrm{O}_{2} \mathrm{c}$ кластером серебра из $\mathrm{AgOO}\{\mathrm{Ce}\} / \mathrm{NP}\{111\}-\mathbf{1 a}^{2}$ описывается реакцией (3), протекающей с потреблением энергии 0.75 эВ:

$$
3 \cdot \mathrm{AgOO}\{\mathrm{Ce}\} / \mathrm{NP}\{111\}-\mathbf{1 a}^{2} \rightarrow \mathrm{OAg}_{3} \mathrm{O}\{\mathrm{Ce}\} / \mathrm{NP}\{100\}-\mathbf{3} \mathbf{a}^{2}+2 \cdot \mathrm{NP}+2 \cdot \mathrm{O}_{2}
$$


Таким образом, данные о стабильности рассмотренных систем с атомами и небольшими кластерами серебра свидетельствуют о предпочтительности денуклеационных преобразований. В соответствии с выводами экспериментальных исследований $[2,7]$ наши данные доказывают то, что поверхностные комплексы изолированных ионов серебра могут быть, главным образом, ответственны за высокую каталитическую активность модифицированного добавлением серебра наноструктурированного оксида $\mathrm{CeO}_{2}$.

\section{Заключение}

Согласно результатам выполненных МФП-расчетов на поверхности модельной наночастицы $\mathrm{Ce}_{21} \mathrm{O}_{42}$ образуются устойчивые комплексы молекулярного кислорода с мономерами, тримерами и тетрамерами серебра с энергиями образования 1.6-4.4 эВ. Ионы церия наночастицы $\mathrm{Ce}_{21} \mathrm{O}_{42}$ вблизи фрагмента $\mathrm{Ag}_{\mathrm{n}}$ оказываются активированными для энергетически предпочтительного взаимодействия с молекулой $\mathrm{O}_{2}$ с образованием супероксидных $\mathrm{Ag}_{\mathrm{n}} \mathrm{OO}\{\mathrm{Ce}\}$-структур. Энергии $\mathrm{E}_{\mathrm{ad}}\left(\mathrm{O}_{2}\right)$ в таких структурах составляют 0.5-1.3 эВ. Связывание молекулы $\mathrm{O}_{2}$ в наиболее низкоэнергетическом комплексе с $\mathrm{Ag} / \mathrm{NP}\{100\}$-адсорбентом рассчитано слабым, $\sim 0.2$ эВ, а в комплексе $\mathrm{O}_{2}$ с $\mathrm{Ag} / \mathrm{NP}\{111\}$-адсорбентом - весьма сильным, $\sim 1.3$ эВ. Последний из этих двух комплексов оказывается более стабильным на 0.2 эВ. Диссоциация молекулы кислорода на атомарно-дисперсированных частицах серебра рассчитана невыгодной.

Образование супероксидных $\mathrm{Ag}_{3} \mathrm{OO}\{\mathrm{Ce}\}-$ и $\mathrm{Ag}_{4} \mathrm{OO}\{\mathrm{Ce}\}$-структур на поверхности нанограни $\{100\}$ рассчитано более предпочтительным, чем на поверхности $\{111\}$ на $\sim 0.3$ эВ. Диссоциативная адсорбция $\mathrm{O}_{2}$ на $\mathrm{Ag}_{3} / \mathrm{NP}$ и $\mathrm{Ag}_{4} / \mathrm{NP}$ оказывается более выгодной, чем молекулярная адсорбция. Энергетический эффект диссоциации $\mathrm{O}_{2}$ составляет 0.5-0.9 эВ для комплексов $\mathrm{Ag}_{3} /$ $\mathrm{NP}$ и 0.6-1.1 эВ для комплексов $\mathrm{Ag}_{4} / \mathrm{NP}$. Барьеры активации диссоциации $\mathrm{O}_{2}$ в $\mathrm{Ag}_{\mathrm{n}} \mathrm{OO}\{\mathrm{Ce}\}-$ комплексах с $\mathrm{n}=3,4$ составляют 1.5-2.1 эВ, что свидетельствует о низких скоростях данных окислительных перегруппировок. Стабильность низкоэнергетических комплексов $\mathrm{O}_{2}$ с $\mathrm{Ag}_{\mathrm{n}}$ / $\mathrm{NP}\{100\}$ и $\mathrm{Ag}_{\mathrm{n}} / \mathrm{NP}\{111\}$ понижается в ряду $\mathrm{AgOO}\{\mathrm{Ce}\} / \mathrm{NP}\{111\}>\mathrm{AgO}_{2} / \mathrm{NP}\{100\}>\mathrm{OAg}_{3} \mathrm{O}\{\mathrm{Ce}\} /$ $\mathrm{NP}\{100\}>\mathrm{OAg}_{4} \mathrm{O}\{\mathrm{Ce}\} / \mathrm{NP}\{100\}>\mathrm{OAg}_{3} \mathrm{O}\{\mathrm{Ce}\} / \mathrm{NP}\{111\}>\mathrm{Ag}_{3} \mathrm{OO}\{\mathrm{Ce}\} / \mathrm{NP}\{100\}>\mathrm{Ag}_{4} \mathrm{OO}\{\mathrm{Ce}\} /$ $\mathrm{NP}\{100\}>\mathrm{OAg}_{4} \mathrm{O} / \mathrm{NP}\{111\}>\mathrm{Ag}_{3} \mathrm{OO}\{\mathrm{Ce}\} / \mathrm{NP}\{111\}>\mathrm{Ag}_{4} \mathrm{OO} / \mathrm{NP}\{111\}$, свидетельствующем о предпочтительности денуклеационного тренда.

\section{Благодарности}

Работа финансово поддержана Комплексной программой фундаментальных исследований Сибирского отделения РАН, проект ІІ.2П/V.44-12. КМН благодарит за финансовую поддержку Европейскую комиссию (FP7 грант ChipCAT № 310191) и Испанское министерство экономики (грант CTQ2015-64618-R, включая FEDER). Авторы благодарят Сибирский суперкомпьютерный центр (г. Новосибирск) и Центр коллективного пользования (г. Красноярск) за предоставленные вычислительные ресурсы.

\section{Список литературы}

1. Aneggi E., Llorca J., de Leitenburg C., Dolcetti G., Trovarelli A. Soot combustion over silversupported catalysts. Appl. Catal. B. 2009. Vol. 91(1-2), P. 489-498. 
2. Sarode P.R., Priolkar K.R., Bera P., Hegde M.S., Emura S., Kumashiro R. Study of local environment of $\mathrm{Ag}$ in $\mathrm{Ag} / \mathrm{CeO}_{2}$ catalyst by EXAFS. Mater. Res. Bull. 2002. Vol. 37(9), P. 1679-1690.

3. Shimizu K., Kawachi H., Satsuma A. Study of active sites and mechanism for soot oxidation by silver-loaded ceria catalyst. Appl. Catal. B. 2010. Vol. 96(1-2), P. 169-175.

4. Hu S., Wang Y., Wang W., Han Y., Fan Q., Feng X., Xu Q., Zhu J. Ag nanoparticles on reducible $\mathrm{CeO}_{2}(111)$ thin films: effect of thickness and stoichiometry of ceria. J. Phys. Chem. C. 2015. Vol. 119(7), P. 3579-3588.

5. Zhang X., Qu Z., Li X., Wen M., Quan X., Ma D., Wu J. Studies of silvet species for lowtemperature $\mathrm{CO}$ oxidation on $\mathrm{Ag} / \mathrm{SiO}_{2}$ catalysts. Separ. Purific. Technol. 2010. Vol. 72(3), P. 395-400.

6. Kundakovic L., Flytzani-Stephanopoulos M. Cu- and Ag-modified cerium oxide catalysts for methane oxidation. J. Catal. 1998. Vol. 179(1), P. 203-221.

7. Bera P., Patil K.C., Hegde M.S. NO reduction, $\mathrm{CO}$ and hydrocarbon oxidation over combustion synthesized $\mathrm{Ag} / \mathrm{CeO}_{2}$ catalyst. Phys. Chem. Chem. Phys. 2000. Vol. 2(16), P. 3715-3719.

8. Rocha T.C.R., Oestereich A., Demidov D.V., Hävecker M., Zafeiratos S., Weinberg G., Bukhtiyarov V.I., Knop-Gericke A., Schlögl R. The silver-oxygen system in catalysis: new insights by near ambient pressure X-ray photoelectron spectroscopy. Phys. Chem. Chem. Phys. 2012. Vol. 14(13), P. 4554-4564.

9. Kibis L.S., Stadnichenko A.I., Pajetnov E.M., Koscheev S.V., Zaykovskii V.I., Boronin A.I. The investigation of oxidized silver nanoparticles prepared by thermal evaporation and radio-frequency sputtering of metallic silver under oxygen. Appl. Surf. Sci. 2010. Vol. 257(2), P. 404-413.

10. Wang J., Liu M., Lin M. Oxygen reduction reactions in the SOFC cathode of $\mathrm{Ag} / \mathrm{CeO}_{2}$. Solid State Ionics. 2006. Vol. 177(9-10), P. 939-947.

11. Machida M., Murata Y., Kishikawa K., Zhang D., Ikeue K. On the reasons for high activity of $\mathrm{CeO}_{2}$ catalyst for soot oxidation. Chem. Mater. 2008. Vol. 20(13), P. 4489-4494.

12. Preda G., Pacchioni G. Formation of oxygen active species in $\mathrm{Ag}$-modified $\mathrm{CeO}_{2}$ catalyst for soot oxidation: A DFT study. Catal. Today. 2011. Vol. 177(1), P. 31-38.

13. Klacar S., Hellman A., Panas I., Grönbeck H. Oxidation of small silver clusters: a density functional theory study. J. Phys. Chem. C. 2010. Vol. 114(29), P. 12610-12617.

14. Bruix A., Lykhach Y., Matolínová I., Neitzel A., Skála T., Tsud N., Vorokhta M., Stetsovych V., Ševčíková K., Mysliveček J., et al. Maximum noble-metal efficiency in catalytic materials: atomically dispersed surface platinum. Angew. Chem. Int. Ed. 2014. Vol. 53(39), P. 10525-10530.

15. Shor A.M., Laletina S.S., Ivanova Shor E.A., Nasluzov V.A., Bukhtiyarov V.I., Rösch N. Interaction of silica-supported small silver clusters with molecular oxygen. A computational study. Surf. Sci. 2014. Vol. 630, P. 265-272.

16. Zhou J., Li Z.-H., Wang W.-N., Fan K.-N. Density functional study of the interaction of molecular oxygen with small neutral and charged silver clusters. Chem. Phys. Lett. 2006. Vol. 421(46), P. 448-452.

17. Liao M.-S., Watts J.D., Huang M.-J. Theoretical comparative study of oxygen adsorption on neutral and anionic $\mathrm{Ag}_{\mathrm{n}}$ and $\mathrm{Au}_{\mathrm{n}}$ clusters $(\mathrm{n}=2-25)$. J. Phys. Chem. C. 2014. Vol. 118(38), P. 2191121927.

18. Luches P., Pagliuca F., Valeri S., Illas F., Preda G., Pacchioni G. Nature of Ag islands and nanoparticles on the $\mathrm{CeO}_{2}$ (111). J. Phys. Chem. C. 2012. Vol. 116(1), P. 1122- 1132. 
19. Krishna K., Bueno-López A., Makkee M., Moulijn J.A. Potential rare earth modified $\mathrm{CeO}_{2}$ catalysts for soot oxidation. Appl. Catal. B. 2007. Vol. 75 (3-4), P. 189-200.

20. Carrettin S., Concepción P., Corma A., López Nieto J.M., Puntes V.F. Nanocrystalline $\mathrm{CeO}_{2}$ increases the activity of $\mathrm{Au}$ for $\mathrm{CO}$ oxidation by two orders of magnitude. Angew. Chem. Int. Ed. 2004. Vol. 43(19), P. 2538-2540.

21. Farmer J.A., Campbell C.T. Ceria maintains smaller metal catalyst particles by strong metalsupport bonding. Science. 2010. Vol. 329(5994), P. 933-936.

22. Chusuei C.C., Lai X., Luo K., Goodman D.W. Modeling heterogeneous catalysts: metal clusters on planar oxide supports. Top. Catal. 2001. Vol. 14(1-4), P. 71-83.

23. Benedetti F., Luches P., Spadaro M.C., Gasperi G., D’Addato S., Valeri S., Boscherini F. Structure and morphology of silver nanoparticles on the (111) surface of cerium oxide. J. Phys. Chem. C. 2015. Vol. 119(11), P. 6024-6032.

24. Chen L.-J., Tang Y., Cui L., Ouyang C., Shi S. Charge transfer and formation of $\mathrm{Ce}^{3+}$ upon adsorption of metal atom $\mathrm{M}(\mathrm{M}=\mathrm{Cu}, \mathrm{Ag}, \mathrm{Au})$ on $\mathrm{CeO}_{2}(100)$ surface. J. Power Sources. 2013. Vol. 234(1), P. 69-81.

25. Branda M.M., Hernández N.C., Sanz J.F., Illas F. Density functional theory study of the interaction of $\mathrm{Cu}, \mathrm{Ag}$, and $\mathrm{Au}$ atoms with the regular $\mathrm{CeO}_{2}(111)$ Surface. J. Phys. Chem. C. 2010. Vol. 114(4), P. 1934-1941.

26. Cui L., Tang Y., Zhang H., Hector L.G., Ouyang C., Shi S., Li H., Chen L. First-principles investigation of transition metal atom $\mathrm{M}(\mathrm{M}=\mathrm{Cu}, \mathrm{Ag}, \mathrm{Au})$ adsorption on $\mathrm{CeO}_{2}(110)$. Phys. Chem. Chem. Phys. 2012. Vol. 14(6), P. 1923.

27. Vayssilov G.N., Migani A., Neyman K. Density functional modeling of the interactions of platinum clusters with $\mathrm{CeO}_{2}$ nanoparticles of different size. J. Phys. Chem. C. 2011. Vol. 115(32), P. 16081-16086.

28. Castellani N.J., Branda M.M., Neyman K.M., Illas F. Density Functional Theory Study of the Adsorption of Au Atom on Cerium Oxide: Effect of Low-Coordinated Surface Sites. J. Phys. Chem. C. 2009. Vol. 113(12), P. 4948-4954.

29. Aleksandrov H.A., Neyman K.M., Vayssilov G.N. Structure and stability of reduced and oxidized mononuclear platinum species on nanostructured ceria from density functional modeling. Phys. Chem. Chem. Phys. 2015. Vol. 17, P. 14551-14560.

30. Shapovalov V., Metiu H. Catalysis by doped oxides: $\mathrm{CO}$ oxidation by $\mathrm{Au}_{\mathrm{x}} \mathrm{Ce}_{1-\mathrm{x}} \mathrm{O}_{2}$. J. Catal. 2007. Vol. 245(1), P. 205-214.

31. Xu J., Harmer J., Li G., Chapman T., Collier P., Longworth S., Tsang S.C. Size dependent oxygen buffering capacity of ceria nanocrystals. Chem. Comm. 2010. Vol. 46(11), P. 1887-1889.

32. Figueroba A., Kovács G., Bruix A., Neyman K.M. Towards stable single-atom catalysts: Strong binding of atomically dispersed transition metals on the surface of nanostructured ceria. Catal. Sci. Technol. 2016. Vol. 6, P. 6806-6813.

33. Bruix A., Neyman K.M. Modeling ceria-based nanomaterials for catalysis and related applications. Catal. Lett. 2016. Vol. 146, P. 2053-2080.

34. Migani A., Vayssilov G.N., Bromley S.T., Illas F., Neyman K.M. Greatly facilitated oxygen vacancy formation in ceria nanocrystallites. Chem. Comm. 2010. Vol. 46(32), P. 5936-5938. 
35. Loschen C., Migani A., Bromley S.T., Illas F., Neyman K.M. Density functional studies of model cerium oxide nanoparticles. Phys. Chem. Chem. Phys. 2008. Vol. 10(37), P. 5730-5738.

36. Preda G., Migani A., Neyman K.M., Bromley S.T., Illas F., Pacchioni G. Formation of superoxide anions on ceria nanoparticles by interaction of molecular oxygen with $\mathrm{Ce}^{3+}$ sites. J. Phys. Chem. C. 2011. Vol. 115(13), P. 5817-5822.

37. Kresse G., Hafner J. Ab initio molecular dynamics for liquid metals. Phys. Rev. B. 1993. Vol. 47(1), P. 558-561.

38. Kresse G., Furthmüller J. Efficient iterative schemes for ab initio total-energy calculations using a plane-wave basis set. Phys. Rev. B. 1996. Vol. 54(16), P. 11169-11186.

39. Blöchl P.E. Projector augmented-wave method. Phys. Rev. B. 1994. Vol. 50(24), P. 1795317979.

40. Kresse G., Joubert D. From ultrasoft pseudopotentials to the projector augmented-wave method. Phys. Rev. B. 1999. Vol. 59(3), P. 1758-1775.

41. Rohrbach A., Hafner J., Kresse G. Electronic correlation effects in transition-metal sulfides. J. Phys.: Condens. Matter. 2003. Vol. 15(6), P. 979-996.

42. Perdew J.P., Chevary J.A., Vosko S.H., Jackson K.A., Pederson M.R., Singh D.J., Fiolhais C. Atoms, molecules, solids, and surfaces: Applications of the generalized gradient approximation for exchange and correlation. Phys. Rev. B. 1992. Vol. 46(11), P. 6671-6687; Erratum. Phys. Rev. B. 1993. Vol. 48(7), P. 4978.

43. Monkhorst H.J., Pack J.D. Special points for Brillouin-zone integrations. Phys. Rev. B. 1976. Vol. 13(12), P. 5188-5192.

44. Chaffey-Millar H., Nikodem A., Matveev A.V., Krüger S., Rösch N. Improving upon string methods for TS discovery. J. Chem. Theor. Comput. 2012. Vol. 8(2), P. 777-786.

45. Zhao Y., Teng B.-T., Wen X.-D., Zhao Y., Chen Q.-P., Zhao L.-H., Luo M.-F. Superoxide and peroxide species on $\mathrm{CeO}_{2}(111)$, and their oxidation roles. J. Phys. Chem. C. 2012. Vol. 116(30), P. 15986-15991. 